\title{
Autofocus and analysis of geometrical errors within the framework of Fast Factorized Back-Projection
}

\author{
Jan Torgrimsson ${ }^{\mathrm{a}}$, Patrik Dammert ${ }^{\mathrm{b}}$, Hans Hellsten ${ }^{\mathrm{b}}$, Lars M. H. Ulander ${ }^{\mathrm{a}, \mathrm{c}}$ \\ ${ }^{a}$ Chalmers University of Technology, Gothenburg, Sweden; ${ }^{\mathrm{b}} \mathrm{SAAB}$ Electronic Defence Systems \\ (EDS), Gothenburg, Sweden; 'S wedish Defence Research Agency (FOI), Linköping, Sweden
}

\begin{abstract}
This paper describes a Fast Factorized Back-Projection (FFBP) formulation that includes a fully integrated autofocus algorithm, i.e. the Factorized Geometrical Autofocus (FGA) algorithm. The base-two factorization is executed in a horizontal plane, using a Merging (M) and a Range History Preserving (RHP) transform. Six parameters are adopted for each sub-aperture pair, i.e. to establish the geometry stage-by-stage via triangles in 3-dimensional space. If the parameters are derived from navigation data, the algorithm is used as a conventional processing chain. If the parameters on the other hand are varied from a certain factorization step and forward, the algorithm is used as a joint image formation and autofocus strategy. By regulating the geometry at multiple resolution levels, challenging defocusing effects, e.g. residual space-variant Range Cell Migration (RCM), can be corrected.

The new formulation also serves another important purpose, i.e. as a parameter characterization scheme. By using the FGA algorithm and its inverse, relations between two arbitrary geometries can be studied, in consequence, this makes it feasible to analyze how errors in navigation data, and topography, affect image focus.

The versatility of the factorization procedure is demonstrated successfully on simulated Synthetic Aperture Radar (SAR) data. This is achieved by introducing different GPS/IMU errors and Focus Target Plane (FTP) deviations prior to processing. The characterization scheme is then employed to evaluate the sensitivity, to determine at what step the autofocus function should be activated, and to decide the number of necessary parameters at each step. Resulting FGA images are also compared to a reference image (processed without errors and autofocus) and to a defocused image (processed without autofocus), i.e. to validate the novel approach further.
\end{abstract}

Keywords: Autofocus, Back-Projection, FFBP, FGA, SAR

\section{INTRODUCTION}

Synthetic Aperture Radar (SAR) is a technology used to form high-resolution imagery ${ }^{1,2}$. The fundamental idea is to synthesize a very long antenna, put into practice by recording radar echoes along a track and combining these coherently. In principle SAR processing resolves the reflectivity of the illuminated scene by focusing raw data. Both time and frequency domain algorithms are available for image formation. Traditionally though, algorithms from the latter category have been favored for faster run time. It is however possible to speed up the standard time domain approach, i.e. Global Back-Projection (GBP) $)^{3,4}$, through factorization. The computational effort of Fast Factorized Back-Projection (FFBP) is comparable to that of range migration processing ${ }^{5-7}$ in the frequency domain. Adding the ability to deal with a nonlinear track, accurately (as opposed to frequency domain algorithms, adopting a local motion compensation scheme), makes this method (FFBP) a viable, or for some scenarios even a superior substitute.

The track is usually measured by means of a GPS and an Inertial Measurement Unit (IMU) ${ }^{1,2}$. The IMU has a high frequency response but drifts over time, hence there is generally a need for GPS support. As the IMU constitutes a major cost and is subject to export restrictions, a desire to relax requirements (or excluding it) often originates. Naturally though, SAR processing relies on measurement accuracy. This of course contradicts the stated desire. A GPS may also be jammed or shadowed, leading to a dependence on the IMU, again affecting the accuracy.

To form a focused image, the track must be measured within fractions $(\sim 1 / 16)$ of a wavelength ${ }^{1,2}$. For high radar bands (e.g. X-band) this demand is typically too strict (to form high-resolution imagery).

Algorithms for Synthetic Aperture Radar Imagery XXI, edited by Edmund Zelnio, Frederick D. Garber, Proc. of SPIE Vol. 9093, $909303 \cdot$ · 2014 SPIE · CCC code: 0277-786X/14/\$18 · doi: 10.1117/12.2050048 
Even for a low radar band such as Very High Frequency (VHF), the issues above may degrade the image. There is however a conceivable solution, making it feasible to focus an image without the otherwise necessary accuracy, viz. autofocus.

In the context of SAR processing, autofocus is the utilization of information in a defocused image (or in the data), to estimate and correct phase errors $\mathrm{s}^{1,2}$. Practical image formation routines normally have autofocus capability, techniques spanning from MapDrift (MD) $)^{1,2}$ and the Phase Gradient Algorithm (PGA) ${ }^{1,2}$, to advanced PGA ${ }^{8}$ PGA-MD $^{9}$ extensions, and multi-lateration methods ${ }^{10,11}$ based on prominent point phase tracking ${ }^{10}$ and 2-dimensional $\mathrm{MD}^{11}$, are in use. The choice of autofocus algorithm is very dependent on measurement accuracy, thus it is important to be able to analyze how (expected) errors in navigation data, and (in addition) topography, affect image focus.

The objective of this paper is to describe and test an FFBP formulation that includes a fully integrated autofocus algorithm, i.e. the Factorized Geometrical Autofocus (FGA) algorithm ${ }^{12}$, also forming the basis for a parameter characterization scheme.

The autofocus strategy (FGA) is in this case also the conventional processing chain (FFBP). This implies that like FFBP, the FGA algorithm can be used to process spotlight or stripmap data. The base-two factorization adopts six tunable parameters for each sub-aperture pair, i.e. to establish the geometry stage-by-stage via triangles in 3-dimensional space. If autofocus is off, parameters are just derived from navigation data. If autofocus on the other hand is on, different geometry hypotheses are assessed by varying the parameters, the aim is then to find the sharpest image according to a chosen object function (intensity correlation). This strategy can compensate for challenging defocusing effects, e.g. residual space-variant Range Cell Migration $(\mathrm{RCM})^{12}$. By using the FGA algorithm and its inverse, it is also possible to study relations between two arbitrary geometries.

The new formulation (and its inverse) has been tried out in spotlight mode on a synthetic Ultra-WideBand (UWB) data set, i.e. with different (simulated) GPS/IMU errors and Focus Target Plane (FTP) deviations introduced prior to processing. An analysis without any image formation is first performed for some basic errors, e.g. a faulty along-track velocity and a faulty acceleration (along-track and cross-track acceleration analyzed separately), i.e. for a linear reference track, and faulty FTPs, i.e. for a nonlinear reference track. An analysis in conjunction with image formation is then performed for a more advanced error, i.e. for a linear reference track.

This paper will present and discuss sensitivity plots from various parameter characterization trials. FGA images will also be shown, and compared to a reference image (processed without errors and autofocus) and to a defocused image (processed without autofocus), suffering from residual space-variant RCM. However, first a review is required, treating time domain SAR processing and of course the FGA concept. The realization and evaluation procedure will be recapped in detail as well.

\section{METHOD}

\subsection{Global Back-Projection}

$\mathrm{GBP}^{3,4}$ is a time domain algorithm, projecting pulse compressed radar echoes from an FTP, to a generally defined Image Display Plane (IDP). Each slow time position (along the track) contributes with a data value to each and every pixel. Complex values are added coherently, causing interference, resolving reflective structures.

Eq. (1) gives a polar GBP expression:

$$
I(\rho, \theta)=\sum_{n=1}^{N} f\left(n, R_{n}\right) \cdot R_{n} \cdot \exp \left(\frac{j 4 \pi R_{n}}{\lambda_{c}}\right)
$$

In (1) $I$ is an image formed from data acquired (or generated) along an arbitrary track (extending across $N$ sample positions, presume that $N$ equals a power of two). 
Each pixel coordinate $(\rho, \theta)$, originates from the centre of a user-defined aperture vector and hits a point in the FTP. The slant range $R_{n}$ between the sample position (with index $n$ ) and the point in question (corresponding to a pixel coordinate), determines which data value $f\left(n, R_{n}\right)$ to accumulate, range interpolation retrieves the proper value from available samples. For demodulated data, each value must also be multiplied by a phase factor, i.e. the exponential with centre wavelength $\lambda_{c}$. The range multiplication is included to establish $1 / R$ dependence (note also that this GBP formulation omits a ramp filter, usually applied to even out the spectrum). In the processing context, customary SAR premises, e.g. the start-stop and the Born approximation, as well as a constant wave velocity, are also adopted ${ }^{4}$.

To summarize; GBP is a versatile algorithm (dealing with nonlinear tracks, topography, etc.), however, the number of operations (proportional to $N^{3}$ for $N$ sample positions and an $N \times N$ image) normally restricts its use to moderately sized images ${ }^{4}$.

Finally it should also be stressed, that it is more common to see Cartesian GBP expressions. The format of (1) is motivated by the fact that this paper in substance describes polar SAR processing.

\subsection{Fast Factorized Back-Projection}

FFBP $^{3,4}$ is a time efficient alternative to GBP, combining pulse compressed radar echoes coherently stage-by-stage.

Consider (1) and the consequence of dividing the sum in (1) into two sums:

$$
I(\rho, \theta)=\sum_{n=1}^{N / 2} f\left(n, R_{n}\right) \cdot R_{n} \cdot \exp \left(\frac{j 4 \pi R_{n}}{\lambda_{c}}\right)+\sum_{n=N / 2+1}^{N} f\left(n, R_{n}\right) \cdot R_{n} \cdot \exp \left(\frac{j 4 \pi R_{n}}{\lambda_{c}}\right)
$$

The sums in (2) may be viewed as low-resolution realizations of $I$. In principle the aperture (the track) is partitioned into sub-apertures, hence the Doppler bandwidth is halved (one half for each sub-aperture). Thus sub-images can be formed with a reduced sampling rate in azimuth (approximately half the original rate), and the addends in (2) can in turn be attained by way of sub-image interpolation.

Eq. (3) gives a polar base-two FFBP expression:

$$
I(\rho, \theta)=I_{1}\left(p_{1}, \theta_{1}\right) \cdot \exp \left(\frac{j 4 \pi \Delta \rho_{1}}{\lambda_{c}}\right)+I_{2}\left(p_{2}, \theta_{2}\right) \cdot \exp \left(\frac{j 4 \pi \Delta \rho_{2}}{\lambda_{c}}\right)
$$

In (3) $I$ is formed by adding a contribution from sub-image $I_{1}$ and $I_{2}$ [corresponding to the addends in (2)]. To elaborate; the pixel coordinate $(\rho, \theta)$, originates from the centre of a user-defined aperture vector and hits a point in the FTP. Subimage coordinates $\left(p_{1}, \theta_{1}\right)$ and $\left(p_{2}, \theta_{2}\right)$ emerge from the sub-apertures (in practice from centres of user-defined subaperture vectors), and converge at this point. Data values $I_{1}\left(p_{1}, \theta_{1}\right)$ and $I_{2}\left(p_{2}, \theta_{2}\right)$ are determined by the coordinates, interpolation (in both range and angle) ${ }^{3,4}$ retrieves proper values from available samples. As in (1), exponentials (with $\Delta \rho_{1}=\rho-\rho_{1}$ and $\left.\Delta \rho_{2}=\rho-\rho_{2}\right)$ compensate for demodulation [note; the phase factors in (3) are only valid for slantbased images].

Essentially, the sum in (1) can be divided into $N / 2$ sums, a multi-step $\left(\log _{2} N\right)$ factorization procedure may then be employed to obtain $I$. The principle is the same as above, the aperture (the track) is partitioned into sub-apertures, increasing in length (finer angular resolution) and decreasing in number for each factorization step ${ }^{4}$. Sub-apertures come with sub-images, i.e. with pixel coordinates in range and sub-lobe angle (basically the antenna beam is split up into sublobes). Ideally, if the number of slow time positions is expressible as a factorization of integers, the final step gives the polar aperture image $I$.

Note that in stripmap mode, the sub-apertures may overlap to smooth transitions between individual aperture images. These are then placed side by side, registered or resampled to a suitable representation (e.g. Cartesian). 
For a base-two implementation, the number of operations is proportional to $2 N^{2} \log _{2} N$ (again for $N$ sample positions and an $N \times N$ image), i.e. under the premise that $N$ equals a power of two ${ }^{4}$. This is a significant reduction in computational effort compared to GBP, equivalent to the one achieved by the Fast Fourier Transform (FFT) ${ }^{13}$ over the Discrete Fourier Transform (DFT) ${ }^{13}$. Image quality requirements may however motivate a less effective algorithm execution (e.g. by reducing the number of steps and/or using a more exact interpolator), to make up for the fact that interpolation errors are accumulated for each factorization step ${ }^{3,4}$.

Finally it should also be stressed, that though this paper only treats base-two factorization, other bases may be outlined in a similar fashion.

\subsection{Factorized Geometrical Autofocus}

The FGA algorithm ${ }^{12}$ will now be described, both in general terms and in detail, the text concentrates on the autofocus function, but applies to conventional (FFBP) processing (autofocus function off) as well.

\section{1) A General Resume}

To set the tone; presume that pulse compressed radar echoes are demodulated and factorized with base-two until two sub-apertures remain. The IDP and the FTP coincide with the horizontal plane or the $x y$-plane. Despite measurement errors (GPS/IMU errors), the sub-images are focused, this due to limited angular resolution. The full aperture is synthesized as a vector $\boldsymbol{Q}_{\mathbf{1 3}}$, extending from the start point $\boldsymbol{p}_{\mathbf{1}}$ of the first sub-aperture $\boldsymbol{Q}_{\mathbf{1 2}}$ to the end point $\boldsymbol{p}_{\mathbf{3}}$ of the other $\boldsymbol{Q}_{\mathbf{2 3}}$. $\boldsymbol{p}_{1}, \boldsymbol{p}_{3}$ and the cut-off point $\boldsymbol{p}_{2}$ between prior vectors form a triangle [see Fig. 1 (left)] or a line as a special case. If the geometry is too erroneous (due to measurement errors), the aperture image will be defocused.

By varying parameters defining the triangle, different geometry hypotheses can be assessed. The variation is carried out consecutively by means of a Merging (M) and a Range History Preserving (RHP) transform. In principle pixel coordinates of the aperture image (i.e. a pixel grid with coordinates in range and lobe angle) are expressed in sub-image coordinates corresponding to the current geometry. The aperture image is then obtained by interpolating the sub-images onto polar pixel grids and adding these coherently. Fundamentally the final factorization step is repeated time after time. Each hypothesis gives an image, which then is marked with a focus measure provided by an object function. The image with the best measure is assumed to be autofocused. Conventional processing on the contrary, just derives the parameters from navigation data, i.e. no variation is carried out. This approach applies up until the final step in the scenario above (note also that in this case the RHP transform is redundant, as it basically becomes a unity transform).

Before proceeding it should be emphasized, that even though one step is considered here, the autofocus function can be activated at any time during the factorization ${ }^{12}$. However, as the demand on measurement accuracy increases quadratically with sub-aperture length, it is more likely that the strategy is required later on in the processing chain.

Note also that this spotlight procedure can deal with stripmap data in the aforementioned way, i.e. by allowing sub-apertures to overlap. For example, instead of forming two images (triangles) from four sub-apertures, three images (triangles) may be formed. The two central sub-apertures are then used to produce the extra image.

Confining the variation to a number of quantities (the fewer the better) is a crucial task. In total, the triangle has nine degrees of freedom (assuming that sub-aperture vectors remain connected in the cut-off point, i.e. an $x, y$ and $z$ coordinate for $\boldsymbol{p}_{1}, \boldsymbol{p}_{2}$ and $\boldsymbol{p}_{3}$ respectively). Translating (two degrees) and rotating (one degree) the triangle horizontally will however only translate and rotate the aperture image. This implies that from a focus perspective, three degrees of freedom can be dropped. Thus the geometry may be described by means of an altitude, three angles and two length variables, all in all six independent parameters ${ }^{12}$.

It should be mentioned, that in an early formulation of this algorithm ${ }^{14}$, the Height Of Focus (HOF) is presumed to suffice, making it possible to fix the position and orientation of one sub-aperture $\left(\boldsymbol{Q}_{\mathbf{1 2}}\right)$ and by that omitting two additional degrees of freedom. 


\section{2) The Algorithm}

Assume once again that the autofocus function is activated at the final factorization step. An arbitrary geometry hypothesis (contrary to the initial geometry hypothesis, specified by means of the measured track) is first expressed in the following quantities [see Fig. 1 (left) for further information]:

- $H_{13}$

- $\phi$

- $\beta_{13}$

- $v$

- $L_{13}$

- $\Delta L$
Altitude at the centre of $\boldsymbol{Q}_{13}$

Angle between $\boldsymbol{\Gamma}$ and $\boldsymbol{\Pi}$

Angle between the $x y$-plane and $\boldsymbol{Q}_{13}$

Angle between $\boldsymbol{Q}_{12}$ and $\boldsymbol{Q}_{23}$

Length of $\boldsymbol{Q}_{13}$

Length difference between $\boldsymbol{Q}_{12}$ and $\boldsymbol{Q}_{23}$

Supporting parameters are computed and the pixel coordinates $\left(\rho_{13}, \theta_{13}\right)$ of the aperture image $I_{13}$ are established. The M-transform then converts these to corresponding sub-image coordinates, i.e. $\left(p_{M 12}, \theta_{M 12}\right)$ and $\left(p_{M 23}, \theta_{M 23}\right)$. This conforms to translating and rotating sub-aperture vectors horizontally, as opposed to translating and rotating an intact triangle. Fig. 1 (right) and (4) to (7) clarify the concept of the M-transform. Note that all parameters in (4) to (7) are defined in the $x y$-plane, $L_{12 x y}$ and $L_{23 x y}$ are horizontal length variables (associated with the sub-apertures), while $v_{x y}$, $\zeta_{x y}$ and $\xi_{x y}$ are projected angles $(v, \zeta$ and $\xi)$.

$$
\begin{gathered}
\rho_{M 12}=\left(\rho_{13}^{2}+\left(L_{23 x y} / 2\right)^{2}-\rho_{13} \cdot L_{23 x y} \cdot \cos \left(\pi-\theta_{13} \pm \xi_{x y}\right)\right)^{1 / 2} \\
\theta_{M 12}=\cos ^{-1}\left(\frac{\rho_{13}^{2}-\rho_{M 12}^{2}-\left(L_{23 x y} / 2\right)^{2}}{-\rho_{M 12} \cdot L_{23 x y}}\right) \pm v_{x y} \\
\rho_{M 23}=\left(\rho_{13}^{2}+\left(L_{12 x y} / 2\right)^{2}-\rho_{13} \cdot L_{12 x y} \cdot \cos \left(\theta_{13} \pm \zeta_{x y}\right)\right)^{1 / 2} \\
\theta_{M 23}=\pi-\cos ^{-1}\left(\frac{\rho_{13}^{2}-\rho_{M 23}^{2}-\left(L_{12 x y} / 2\right)^{2}}{-\rho_{M 23} \cdot L_{12 x y}}\right) \pm v_{x y}
\end{gathered}
$$

M-transformed pixel coordinates are distorted by the RHP-transform. This conforms to tilt, altitude and length alterations. Contrary to the M-transform, the RHP-transform works with individual sub-aperture vectors (note that subscripts in (8) to (14) below are dropped for clarity, i.e. either 12 or 23 depending on the sub-aperture under consideration).

Consider $\boldsymbol{Q}_{\mathbf{0}}$, defined between times: $-T / 2<t<T / 2$ (the zero subscript signifies that $\boldsymbol{Q}_{\mathbf{0}}$ is specified by means of the measured track). The slant range to the point $\boldsymbol{P}$ along $\boldsymbol{Q}_{\mathbf{0}}$ as a function of time is given by:

$$
\left\|\boldsymbol{Q}_{0}(t) \boldsymbol{P}\right\|=\left(\left(\rho_{M} \cdot \sin \theta_{M}\right)^{2}+\left(\rho_{M} \cdot \cos \theta_{M}-V_{0 x y} t\right)^{2}+\left(H_{0}+V_{0 z} t\right)^{2}\right)^{1 / 2}
$$

In (8) $\rho_{M}$ is the range, while $\theta_{M}$ is the sub-lobe angle (in the $x y$-plane). $H_{0}$ is the altitude at the centre of the sub-aperture vector (defined at time zero just as $\rho_{M}$ and $\theta_{M}$ ), $V_{0 x y}$ is the horizontal speed and $V_{0 z}$ is the vertical velocity.

Now consider $\boldsymbol{Q}$, defined between the same times as before, but with altered horizontal speed $V_{x y}$, vertical velocity $V_{z}$ and altitude $H$. The slant range to the point $\boldsymbol{P}$ along $\boldsymbol{Q}$ as a function of time is given by:

$$
\|\boldsymbol{Q}(t) \boldsymbol{P}\|=\left(\left(\rho_{M} \cdot \sin \theta_{M}\right)^{2}+\left(\rho_{M} \cdot \cos \theta_{M}-V_{x y} t\right)^{2}+\left(H+V_{z} t\right)^{2}\right)^{1 / 2}
$$



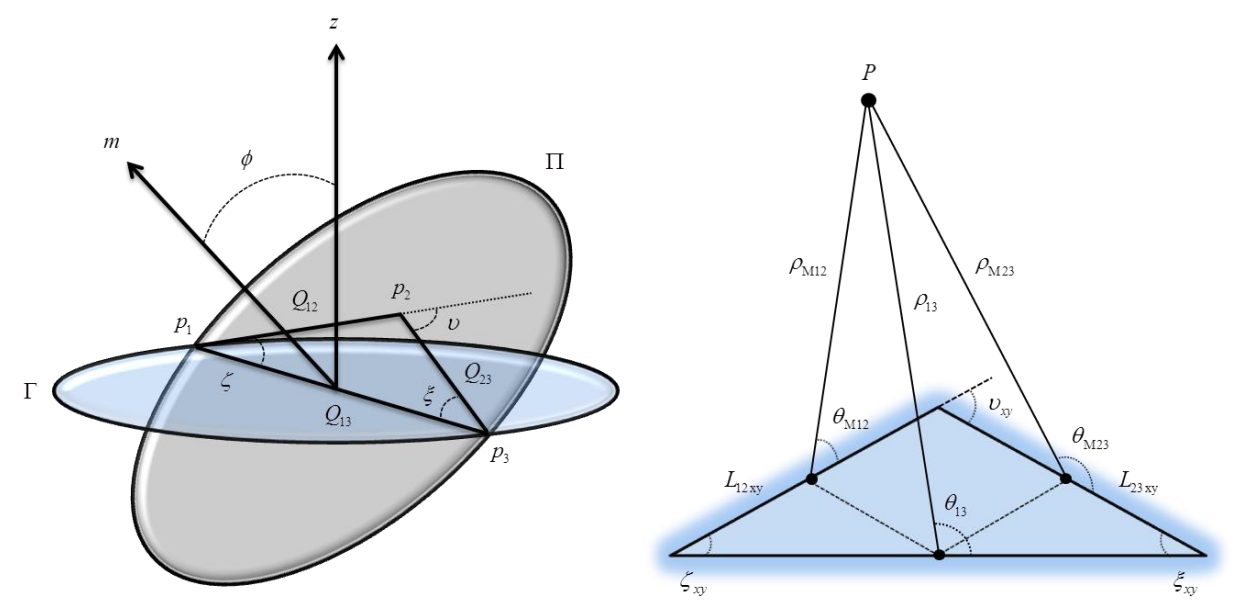

Figure 1 (left) Shows the triangle in the plane $\boldsymbol{\Pi}$ (grey). $\phi, v, \zeta$ and $\xi$ are essential parameters in this algorithm. Note that $\boldsymbol{Q}_{\mathbf{1 3}}$ and a horizontal vector orthogonal to $\boldsymbol{Q}_{\mathbf{1 3}}$ (not shown) define the plane $\boldsymbol{\Gamma}$ (blue). In this particular case $\boldsymbol{Q}_{\mathbf{1 3}}$ and $\boldsymbol{\Gamma}$ coincide with the xyplane (blue). (right) Shows the geometry for the M-transform (i.e. the triangle to the left, projected to the xy-plane). The point $\boldsymbol{P}$ is an arbitrary point in the IDP/FTP. In practice $\boldsymbol{P}$ and the vertex of the cut-off point can be located on either side of $\boldsymbol{Q}_{13}$, explaining the \pm signs in (4) to (7).

Note that though $V$ components are constants in (8) and (9), this is not a limiting requirement (i.e. the velocity is allowed to vary along the track), but a way to clarify the coming derivation. In practice $V$ components below are replaced by length $(L)$ components.

The RHP-transform substitutes $\rho_{M}$ and $\theta_{M}$ in (8) with primed parameters, i.e. $\rho_{M}^{\prime}$ and $\theta_{M}^{\prime}$. The goal is to find equality or at least approximate equality between the range histories of $\|\boldsymbol{Q}(t) \boldsymbol{P}\|$ and $\left\|\boldsymbol{Q}_{\mathbf{0}}^{\prime}(t) \boldsymbol{P}\right\|$. This is realized by first squaring and expanding (9) and the primed (8). Resulting polynomial coefficients $(t)$ are then set to agree:

$$
\rho_{M}^{\prime 2}+H_{0}^{2}=\rho_{M}^{2}+H^{2}
$$

giving:

$$
\rho_{M}^{\prime}=\sqrt{\left(\rho_{M}^{2}+H^{2}-H_{0}^{2}\right)}
$$

and

$$
\left(\rho_{M}^{\prime} \cdot V_{0 x y} \cdot \cos \theta_{M}^{\prime}-H_{0} \cdot V_{0 z}\right) t=\left(\rho_{M} \cdot V_{x y} \cdot \cos \theta_{M}-H \cdot V_{z}\right) t
$$

giving:

$$
\theta_{M}^{\prime}=\cos ^{-1}\left(\frac{\rho_{M} \cdot V_{x y} \cdot \cos \theta_{M}-H \cdot V_{z}+H_{0} \cdot V_{0 z}}{\rho_{M}^{\prime} \cdot V_{0 x y}}\right)
$$

and

$$
\left(V_{0 x y}^{2}+V_{0 z}^{2}\right) t^{2}=\left(V_{x y}^{2}+V_{z}^{2}\right) t^{2}
$$

The second order equality in (14) is only satisfied if the original sub-aperture length (given by the initial geometry hypothesis) is preserved while varying the geometry. However, if the sub-images are focused as assumed, second order equality is not required, as it is possible to compensate for errors in navigation data with the aid of (11) and (13) alone. 
The sub-images are interpolated onto polar pixel grids with M and RHP-transformed coordinates (corresponding to the current geometry, i.e. the arbitrary geometry hypothesis). Adding the grids coherently as in (15) then gives the aperture image. Note that phase factors (demodulated data) are omitted for clarity.

$$
I_{13}\left(\rho_{13}, \theta_{13}\right)=I_{12}\left(\rho_{M 12}^{\prime}, \theta_{M 12}^{\prime}\right)+I_{23}\left(\rho_{M 23}^{\prime}, \theta_{M 23}^{\prime}\right)
$$

To decide if the focus level is satisfactory, the grid similarity is calculated through intensity correlation, an object function also employed in the MD technique mentioned earlier. If the normalized correlation sum in (16) is adequately close to unity, the aperture image is autofocused, if not, another geometry hypothesis must be assessed.

$$
C=\frac{\sum \sum\left(g_{12}-m_{12}\right) \cdot\left(g_{23}-m_{23}\right)}{\sqrt{\left(\sum \sum\left(g_{12}-m_{12}\right)^{2}\right) \cdot\left(\sum \sum\left(g_{23}-m_{23}\right)^{2}\right)}}
$$

In (16) $g_{12}=\left|I_{12}\left(\rho_{M 12}^{\prime}, \theta_{M 12}^{\prime}\right)\right|^{2}$ and $g_{23}=\left|I_{23}\left(\rho_{M 23}^{\prime}, \theta_{M 23}^{\prime}\right)\right|^{2}$ are magnitude squared grids, while $m_{12}$ and $m_{23}$ are corresponding average values. Note also that the summation is carried out over all pixels.

An exhaustive 6-dimensional geometry search is not a feasible approach in practice, especially not if several autofocus steps (factorization steps with adjustable parameters) are required, i.e. if the autofocus function is activated early on in the processing chain ${ }^{12}$. However, for a near-linear aperture (track), the sensitivity is low for $H_{13}, \phi$ and $\beta_{13}$ (external triangle quantities), potentially limiting the FGA algorithm to a variation of $v, L_{13}$ and $\Delta L$ (internal triangle quantities). Naturally this facilitates the autofocus problem. If the size of the scene is restricted as well, it may even be viable to vary a single parameter, such as $v$. In general though, the premises above cannot be taken for granted, as the number of quantities needed to retrieve a focused image is influenced by wavelength, bandwidth, resolution, measurement accuracy, scene size, etc. Thus the strategy must be able to correct six parameters if necessary.

\subsection{Parameter Characterization}

It is evident, that if $\mathrm{M}$ and RHP-transformed coordinates are inserted into an Inverse RHP-transform (IRHP), i.e. (17) and (18), M-transformed coordinates will result, i.e. $\left(p_{M 12}, \theta_{M 12}\right)$ and $\left(p_{M 23}, \theta_{M 23}\right)$.

$$
\begin{gathered}
\rho_{M}=\sqrt{\left(\rho_{M}^{\prime 2}-H^{2}+H_{0}^{2}\right)} \\
\theta_{M}=\cos ^{-1}\left(\frac{\rho_{M}^{\prime} \cdot V_{0 x y} \cdot \cos \theta_{M}^{\prime}+H \cdot V_{z}-H_{0} \cdot V_{0 z}}{\rho_{M} \cdot V_{x y}}\right)
\end{gathered}
$$

It is also evident, that if M-transformed coordinates are inserted into an Inverse M-transform (IM), i.e. (19) to (22), two sets of original pixel coordinates $\left(\rho_{13}, \theta_{13}\right)$ will result (one set for each sub-aperture, told apart by an extra subscript, i.e. either 12 or 23 ), these sets will of course be identical, as the geometry hypothesis is the same in the forward, and in the inverse algorithm.

$$
\begin{gathered}
\rho_{13(12)}=\left(\cos \left(\theta_{\mathrm{M} 12} \pm \mathrm{v}_{\mathrm{xy}}\right) \cdot\left(-\rho_{M 12} \cdot L_{23 x y}\right)+\rho_{M 12}^{2}+\left(L_{23 x y} / 2\right)^{2}\right)^{1 / 2} \\
\theta_{13(12)}=\pi-\cos ^{-1}\left(\frac{\rho_{M 12}^{2}-\rho_{13(12)}^{2}-\left(L_{23 x y} / 2\right)^{2}}{-\rho_{13(12)} \cdot L_{23 x y}}\right) \pm \xi_{x y} \\
\rho_{13(23)}=\left(\cos \left(\pi \pm v_{\mathrm{xy}}-\theta_{\mathrm{M} 23}\right) \cdot\left(-\rho_{M 23} \cdot L_{12 x y}\right)+\rho_{M 23}^{2}+\left(L_{12 x y} / 2\right)^{2}\right)^{1 / 2} \\
\theta_{13(23)}=\cos ^{-1}\left(\frac{\rho_{M 23}^{2}-\rho_{13(23)}^{2}-\left(L_{12 x y} / 2\right)^{2}}{-\rho_{13(23)} \cdot L_{12 x y}}\right) \pm \varsigma_{x y}
\end{gathered}
$$




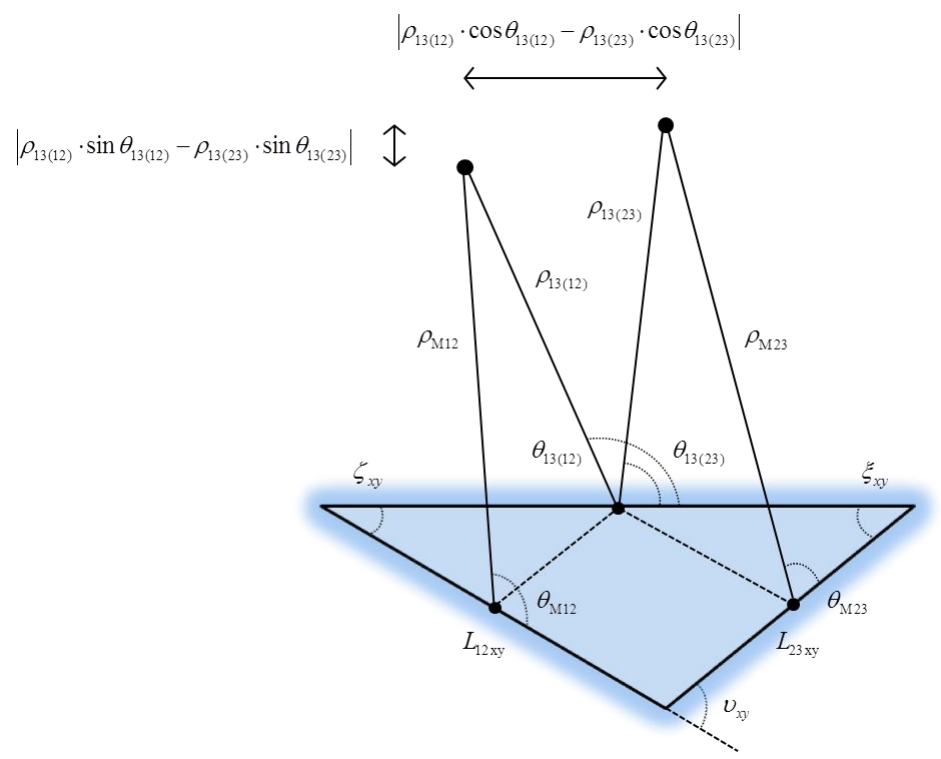

Figure 2 Shows a case where the geometry has been updated prior to inverse transformation, giving different sets and hence a coordinate shift. In processing terms, this implies that erroneous data values will be retrieved when interpolating, possibly producing a defocused image, i.e. depending on the magnitude of the shift.

Now, if the hypothesis under consideration is updated prior to inverse transformation (IRHP and IM); the sets will not necessarily be identical (see Fig. 2). The shift between corresponding coordinates (in the two sets) may be computed in two dimensions, e.g. azimuth and range. By using the FGA algorithm and its inverse in this fashion, relations between two arbitrary geometries can be studied (i.e. parameter characterization). Logically, the true track (synthetic data), the measured track (real data, off-line processing) or a model of it (real data, real-time processing) represents the first geometry, the second is the first with (expected) measurement errors and/or FTP deviations added. Fundamentally, the shift gives a direct indication of the focus level in an aperture image or in a sub-image, formed with erroneous navigation data and/or an FTP offset. In (23) and (24), the absolute shift in azimuth and range is calculated and normalized by half a resolution cell (in azimuth $\delta_{\alpha}$ and range $\delta_{\rho}$ respectively).

$$
\begin{gathered}
\varepsilon_{\alpha}=\frac{\left|\rho_{13(12)} \cdot \cos \theta_{13(12)}-\rho_{13(23)} \cdot \cos \theta_{13(23)}\right|}{\left(\delta_{\alpha} / 2\right)} \\
\varepsilon_{\rho}=\frac{\left|\rho_{13(12)}-\rho_{13(23)}\right|}{\left(\delta_{\rho} / 2\right)}
\end{gathered}
$$

If either $\varepsilon_{\alpha}$ or $\varepsilon_{\rho}$ exceeds unity (focus criteria), the aperture image (or the sub-image) will be defocused. However, for a realistic scenario, it is often sufficient to confirm that $\varepsilon_{\alpha}$ has a value lower than one (for all coordinates of interest), hence this paper only presents sensitivity plots based on (23).

\subsection{The Data Set}

The FGA algorithm (and its inverse) has been applied on a noise-free, synthetic UWB data set made up of 21 point targets, arranged in an ordered structure across a flat, one square km large scene. A CARABAS $\mathrm{II}^{15}$ like system generates the data in stripmap mode, i.e. for different tracks, measurement errors (GPS/IMU errors) and FTP deviations (to support the coming analysis). However, as radar echoes from a limited number of sample positions are made available (and processed), the integration angle (and the resolution) varies across the scene as in spotlight mode. Table 1 resumes data related information (system and geometry quantities). 
Table 1 resumes system and geometry quantities. The size of the scene is rather restricted to reduce run time and save memory. The sample spacing is reported for raw data, not image data, and the squint angle $\left(0^{\circ}\right)$ implies broadside imaging.

\begin{tabular}{l|r}
\hline Quantity & Value \\
\hline System centre frequency & $55 \mathrm{MHz}$ \\
System bandwidth & $70 \mathrm{MHz}$ \\
Integration time & $20 \mathrm{~s}$ \\
Integration angle & $45^{\circ}-75^{\circ}$ \\
Squint angle & $0^{\circ}$ \\
Azimuth spacing & $0.49 \mathrm{~m}$ \\
Slant range spacing & $0.82 \mathrm{~m}$ \\
Slant range (scene centre) & $1800 \mathrm{~m}$ \\
Size of scene & $2000 \mathrm{~m}$ \\
Nominal aperture length & $750 \mathrm{~m}$ \\
Nominal altitude & 21 \\
Number of targets &
\end{tabular}

\subsection{Realization \& Evaluation}

The first task is to perform an analysis for some basic errors, e.g. a faulty along-track velocity and a faulty acceleration (along-track and cross-track acceleration analyzed separately), i.e. for a linear reference track at constant altitude, and faulty FTPs, i.e. for a nonlinear reference track (also at constant altitude). This is realized by specifying true tracks, measurement errors and FTP deviations. Eq. (23) is then used for each (true/false) case, to compute $\varepsilon_{\alpha}$ for polar coordinates (range and cosine of lobe angle in the $x y$-plane) covering the scene described in Table 1. $\varepsilon_{\alpha}$-results are finally plotted (sensitivity plots), revealing focus levels in aperture images (not actually formed).

The second task is to perform an analysis for a more advanced error, i.e. a faulty $x y z$ acceleration (an along-track, a cross-track and a vertical acceleration component of equal magnitude) superposed on a linear reference track at constant altitude. This is realized by specifying a true track and measurement errors. Eq. (23) is then used to compute $\varepsilon_{\alpha}$ for polar coordinates (range and cosine of lobe/sub-lobe angle in the $x y$-plane) covering the scene described in Table 1. $\varepsilon_{\alpha}$-results are finally plotted (sensitivity plots), revealing focus levels in the aperture image, and in the sub-images.

Sensitivity plots down to the first factorization step where all $\varepsilon_{\alpha}$-values are below unity (for all sub-images at that step) are produced, at this step, the autofocus function must be activated to eventually (ideally) retrieve a focused image (in principle, sensitivity plots for preceding steps should be evaluated as well, to really assure that the autofocus function is not activated too late).

The number of necessary parameters at each autofocus step can be determined by replacing parameters for the false track with various parameter combinations (the number of parameters, which parameters and ultimately their values, should be considered), until the sensitivity plots produced by the characterization scheme are satisfying (for all sub-images at the step under consideration). This is an optimization problem that can be solved by means of a Broyden-Fletcher-GoldfarbShanno (BFGS) ${ }^{16}$ search (or some other fast search method) in parameter space, i.e. minimization with respect to minima in the plots. Note that this approach is approximate for later autofocus steps, as the geometry modification of the first step alters the prerequisites.

Three autofocus steps are required to correct the faulty $x y z$ acceleration, one parameter $\left(L_{13}\right)$ is enough for the first step, while three parameters $\left(L_{13}, v\right.$ and $\left.\Delta L\right)$ suffice for the remaining steps. 
Eight (low-resolution) sub-images are first formed by way of GBP, i.e. by employing (1) with limits: $n=1 \rightarrow N / 8$, $n=N / 8+1 \rightarrow N / 4$, etc. [i.e. the aperture (the track) is partitioned into adjacent sub-apertures]. Complex $f\left(n, R_{n}\right)$ values are found through nearest neighbor interpolation of discrete (in range as well) radar echoes $f\left(n, R_{d}\right)$, upsampled eight times in slant range (giving a sample spacing about 21 times finer than Nyquist in range, due to an initial oversampling factor). The FGA algorithm (autofocus function on) is then executed in three steps, both with a reduced parameter set (see above) and with the full set (i.e. all six parameters). Sub-apertures are factorized with 2-dimensional cubic spline interpolation, while the geometry search is accomplished by means of a BFGS method, i.e. minimization with respect to one minus the normalized correlation sum in (16).

After the final FGA image has been obtained, a polar to Cartesian conversion is carried out (again with 2-dimensional cubic spline interpolation), giving an azimuth and slant range representation, contrary to a polar representation in the $x y$ plane. A ramp filter is then applied in the 2-dimensional frequency domain, before finally taking resolution (3-dB in azimuth and slant range) and Peak to SideLobe Ratio (PSLR) measurements. Note also that prior to measuring, target chips are extracted and upsampled 50 times (by way of zero padding in the 2-dimensional frequency domain).

Apart from FGA images; a reference image (processed without errors and autofocus) and a defocused image (processed without autofocus) are also produced; note that the post-processing procedure is the same as above. The complete collection enables a comprehensive error analysis.

\section{RESULTS}

Results will now be presented figure by figure, starting with the basic error analysis; The top-left plot in Fig. 3 shows the sensitivity characteristics for an along-track velocity error $\left(\Delta v_{x}=+0.052 \mathrm{~m} / \mathrm{s}\right)$ at the final factorization step; a linear reference track at constant altitude is presumed from now on [with (Cartesian) velocity $(v)$, acceleration $(a)$ and altitude (h): $v_{x}=100 \mathrm{~m} / \mathrm{s}, v_{y}=v_{z}=0 \mathrm{~m} / \mathrm{s}, a_{x}=a_{y}=a_{z}=0 \mathrm{~m} / \mathrm{s}^{2}$ and $\left.h=750 \mathrm{~m}\right]$. The time base is symmetric (i.e. the aperture is defined between times: $-\tau / 2<t<\tau / 2$ ), the result would however be the same for an asymmetric time base $(0<t<\tau)$. Note also that the velocity error has been chosen to make the $\varepsilon_{\alpha}$-value at scene centre equal to unity and that some near-range values are not acceptable from a focus perspective (as $\varepsilon_{\alpha}$ exceeds unity). In addition, it should be stressed, that the sign of the error does not affect the general properties of the plot, apart from minor $\varepsilon_{\alpha}$ alterations.

The mid-left plot in Fig. 3 shows the sensitivity characteristics for an along-track acceleration error $\left(\Delta a_{x}=+0.0052\right.$ $\mathrm{m} / \mathrm{s}^{2}$ ) at the final factorization step. The time base is asymmetric and the error has once again been chosen to make the $\varepsilon_{\alpha}$-value at scene centre equal to unity. Naturally this plot does not display a symmetrical response, as the error effect is different for the two sub-apertures (that are to be merged into an aperture). The bottom-left plot in Fig. 3 shows the corresponding sensitivity characteristics for a symmetric time base, actually giving a symmetrical response. In addition, it should be stressed again, that the sign of the error does not influence general properties of the plots, i.e. apart from minor $\varepsilon_{\alpha}$ alterations.

The top-right plot in Fig. 3 shows the sensitivity characteristics for a cross-track acceleration error $\left(\Delta a_{y}=+0.0063\right.$ $\mathrm{m} / \mathrm{s}^{2}$ ) at the final factorization step. The time base is symmetric, the result would however be similar for an asymmetric time base, with the exception of small changes in $\varepsilon_{\alpha}$ and a translation of the plot in the azimuth (cosine of lobe angle in the $x y$-plane). Note also that the acceleration error has been chosen to make the $\varepsilon_{\alpha}$-value at scene centre equal to unity and that some far-range values are not acceptable from a focus perspective. In addition, it should be stressed, that the sign of the error does not affect the general properties of the plot, apart from minor $\varepsilon_{\alpha}$ alterations.

The mid-right plot in Fig. 3 shows the sensitivity characteristics for a positive FTP offset $(\Delta h=+150 \mathrm{~m})$ at the final factorization step; a nonlinear reference track (generated with $a_{y}=0.1575 \mathrm{~m} / \mathrm{s}^{2}$ and a symmetric time base) at constant altitude is presumed. Fundamentally the plot reveals that the focus level in the aperture image improves with increasing range, or equivalent, that the HOF augments with range. The bottom-right plot in Fig. 3 shows the corresponding sensitivity characteristics for a negative FTP offset $(\Delta h=-150 \mathrm{~m})$, giving similar plot properties, with the exception of changes in $\varepsilon_{\alpha}$ and in the curvature. Two white areas are also seen, where (19) to (22) have rendered at least one complex solution and in consequence erroneous $\varepsilon_{\alpha}$-values. 
Proceeding with the advanced error analysis; Fig. 4 shows sensitivity plots for an autofocus problem where a faulty $x y z$ acceleration $\left(\Delta a_{x}=\Delta a_{y}=\Delta a_{z}=+0.05 \mathrm{~m} / \mathrm{s}^{2}\right)$ is superposed on a linear reference track in the $x y$-plane (i.e. the reference track specified in the beginning of this section); the time base is asymmetric. Results for the aperture, the first two sub-apertures, and the four sub-apertures of the preceding step demonstrate a focus level degradation with time as expected, i.e. the error effect is larger for sub-apertures representing latter parts of the track. Impairment with increasing sub-aperture length (finer angular resolution) is also observed. The bottom-right plot in Fig. 4 shows the sensitivity characteristics for the last (the eighth out of eight, i.e. the worst case) sub-aperture, at the first factorization step where all $\varepsilon_{\alpha}$-values are below unity.

The top-left plot in Fig. 5 shows the (focused) reference image for the scenario just discussed. The mid-top-left plot shows the defocused image, displaying residual space-variant RCM (curved targets are not shaped the same). The midbottom-left plot shows an autofocused (FGA) image, processed in three steps with the full parameter set. It is apparent that the scene in this case has been translated, rotated and distorted. However, measurements (3-dB in azimuth and slant range, and PSLR) on 18 targets (out of 21, the ones visible in the image) disclose that resolution and PSLR are still close to corresponding reference values (and to theoretical ${ }^{17,18}$ values), i.e. within $4 \%$ and $0.4 \mathrm{~dB}$. The bottom-left plot also shows an FGA image, processed in three steps with a reduced parameter set (see sub-section 2.6). Measurements on all 21 targets disclose that resolution and PSLR are very close to reference values, i.e. within $1 \%$ and $0.1 \mathrm{~dB}$. The close-ups on the right side in Fig. 5 clarify the results further.

Before moving on, it should be stated, that just as above, a number of autofocus problems (also involving noise-free, synthetic UWB data, generated in stripmap mode, i.e. for both linear and nonlinear reference tracks, and various types of advanced errors) have been solved successfully by applying the FGA algorithm (and its inverse). One exception has been encountered though; this (among other things) is discussed briefly below.

\section{DISCUSSION}

The sensitivity plots in Fig. 3 show examples of how basic errors influence the focus level in the final aperture image. In this context, a faulty cross-track velocity, a faulty vertical velocity and a faulty vertical acceleration have been omitted in the analysis, as the corresponding results would be similar to the result for an along-track velocity error (see top-left plot in Fig. 3). Furthermore, the top-left and the top-right plot are complementary, i.e. an error in $L_{13}$ gives a focus level deterioration with decreasing range, while an error in $v_{x y}$ gives a focus level deterioration with increasing range.

It should also be stressed (again), that even if all $\varepsilon_{\alpha}$-values at a certain factorization step are below unity (for all subimages at that step), there is no guarantee that all $\varepsilon_{\alpha}$-values earlier on in the processing chain are lower than one.

The autofocus results are satisfying; target measurements confirm that the FGA algorithm can compensate for residual space-variant RCM. It is interesting that the FGA image processed with fewer parameters is sharper than the one processed with the full parameter set. The reason for this is twofold. First: more parameters imply more local minima and hence a higher risk for the BFGS method to find a sub-optimal solution. Second: as targets are moved out of the area being imaged (due to a rather extreme geometry hypothesis), numerical instabilities (may) arise; this can in principle be prevented by restraining the search space. Local minima are however hard to avoid, especially if noise is present as well, which of course always is the case when it comes to real data. In fact, for another autofocus problem, the FGA algorithm did actually produce a defocused image when adopting all six parameters in the geometry search, due to getting stuck in a very shallow local minimum (for this problem no targets were moved out of the image area either). When limiting the number of quantities though, a focused image was formed. Thus there is a motivation to use as few parameters as possible to establish the new geometry, not only to reduce the run time, but also to obtain a sharper image.

The object function is also an important subject to address. Intensity correlation has worked well thus far; this has been demonstrated for both synthetic and real CARABAS II $^{15}$ data sets (constrained tests ${ }^{12}$ on real data). There is however a concern, as the correlation assumes that targets look the same from different aspect angles. This is in general not true; especially not for UWB systems with a wide antenna beam (e.g. CARABAS II). In consequence, alternative object functions should be considered as well. 


\section{CONCLUSIONS}

We have described a novel FFBP formulation that includes a fully integrated autofocus algorithm, i.e. the FGA algorithm, also forming the basis for a parameter characterization scheme. In substance; the strategy can be used in three ways. First: as a conventional, base-two (FFBP) processing chain (autofocus function off). Second: as an innovative factorization procedure, regulating the geometry by way of six (or fewer if viable) independent parameters, i.e. stage-bystage adjustment, for each sub-aperture pair, or in other words FGA (autofocus function on). Third: as a tool for studying relations between two arbitrary geometries (i.e. parameter characterization). Essentially, the FGA algorithm and its inverse examine two disparate tracks, making it feasible to analyze how errors in navigation data, and topography, affect image focus (at multiple resolution levels). In consequence, the characterization scheme can be utilized to determine the number of autofocus steps required, the number of necessary parameters at each step and which parameters, i.e. for a given (expected) error scenario.

The abilities of the algorithm (and its inverse) are demonstrated on simulated SAR data, i.e. generated for different tracks, GPS/IMU errors and FTP deviations. Sensitivity plots are presented and an autofocus problem is solved with satisfying results. Resolution and PSLR measurements on point targets in FGA images and in a reference image, give approximately the same values (within a few percents and tenths of a dB), supporting the statement that the strategy can correct residual space-variant RCM. The geometry search is, as mentioned, accomplished by means of a BFGS method. This is a very fast approach compared to an exhaustive search routine. However, we conclude that it is not reliable enough (see section 4, third paragraph), hence a more robust method should be developed before testing the algorithm further on real data.

\section{ACKNOWLEDGMENT}

This work was funded by the Swedish Governmental Agency for Innovation Systems (VINNOVA). 


\section{REFERENCES}

[1] Carrara, W. G., Goodman, R. S. and Majewski, R. M., [Spotlight Synthetic Aperture Radar: Signal Processing Algorithms], Artech House Inc, Norwood, MA, (1995).

[2] Jakowatz, C. V., Wahl, D. E., Eichel, P. H., Ghiglia, D. C. and Thompson, P. A., [Spotlight-Mode Synthetic Aperture Radar: A Signal Processing Approach], Kluwer Academic Publishers, (1996).

[3] Frölind, P.-O. and Ulander, L. M. H., "Evaluation of angular interpolation kernels in fast factorized backprojection," Proc. of IEE Radar, Sonar and Navigation 153(3), 243-249 (2006).

[4] Ulander, L. M. H., Hellsten, H. and Stenström, G., "Synthetic aperture radar processing using fast factorized backprojection," IEEE Transactions on Aerospace and Electronic Systems 39(3), 760-776 (2003).

[5] Cafforio, C., Prati, C. and Rocca, F., "SAR data focusing using seismic migration techniques," IEEE Transactions on Aerospace and Electronic Systems 27(2), 194-207 (1991).

[6] Reigber, A., Alivizatos, E., Potsis, A. and Moreira, A., "Extended wavenumber-domain synthetic aperture radar focusing with integrated motion compensation," Proc. of IEE Radar, Sonar and Navigation 153(3), 301-310 (2006).

[7] Rocca, F., Cafforio, C. and Prati, F., "Synthetic aperture radar: a new application for wave equation techniques," Geophysical Prospecting 37, 809-830 (1989).

[8] Doerry, A. W., "Autofocus correction of SAR images exhibiting excessive residual migration," Proc. of SPIE Radar Sensor Technology IX 5788, 34-45 (2005).

[9] Zhu, D., Jiang, R., Mao, X. and Zhu, Z., "Multi-subaperture PGA for SAR autofocusing," IEEE Transactions on Aerospace and Electronic Systems 49(1), 468-488 (2013).

[10]Cantalloube, H. and Dubois-Fernandez, P., "Airborne X-band SAR imaging with $10 \mathrm{~cm}$ resolution: technical challenge and preliminary results," Proc. of IEE Radar, Sonar and Navigation 153(2), 163-176 (2006).

[11] Cantalloube, H. and Nahum, C. E., "Multiscale local map-drift driven multilateration SAR autofocus using fast polar format image synthesis," IEEE Transactions on Geoscience and Remote Sensing 49(10), 3730-3736 (2011).

[12] Torgrimsson, J., Dammert, P., Hellsten, H. and Ulander, L. M. H., "Factorized geometrical autofocus for synthetic aperture radar processing," IEEE Transactions on Geoscience and Remote Sensing (accepted for publication), (2014).

[13] Proakis, J. G. and Manolakis, D. G., [Digital Signal Processing: Principles, Algorithms, and Applications, $4^{\text {th }}$ Edition], Pearson Prentice Hall, Upper Saddle River, New Jersey, (2007).

[14] Hellsten, H., Dammert, P. and Åhlander, A., "Autofocus in fast factorized backprojection for processing of SAR images when geometry parameters are unknown," Proc. of IEEE Radar Conference, 603-608 (2010).

[15] Hellsten, H., Ulander, L. M. H., Gustavsson, A. and Larsson, B., "Development of VHF CARABAS II SAR," Proc. of SPIE Radar Sensor Technology 2747, 48-60 (1996).

[16] Nocedal, J. and Wright, S. J., [Numerical Optimization], Springer, New York, (2006).

[17] Vu, V. T., Sjögren, T. K., Pettersson, M. I. and Hellsten, H., "An impulse response function for evaluation of ultrawideband SAR imaging," IEEE Transactions on Signal Processing 58(7), 3927-3932 (2010).

[18] Vu, V. T., Sjögren, T. K. and Pettersson, M. I., "On synthetic aperture radar azimuth and range resolution equations," IEEE Transactions on Aerospace and Electronic Systems 48(2), 1764-1769 (2012). 

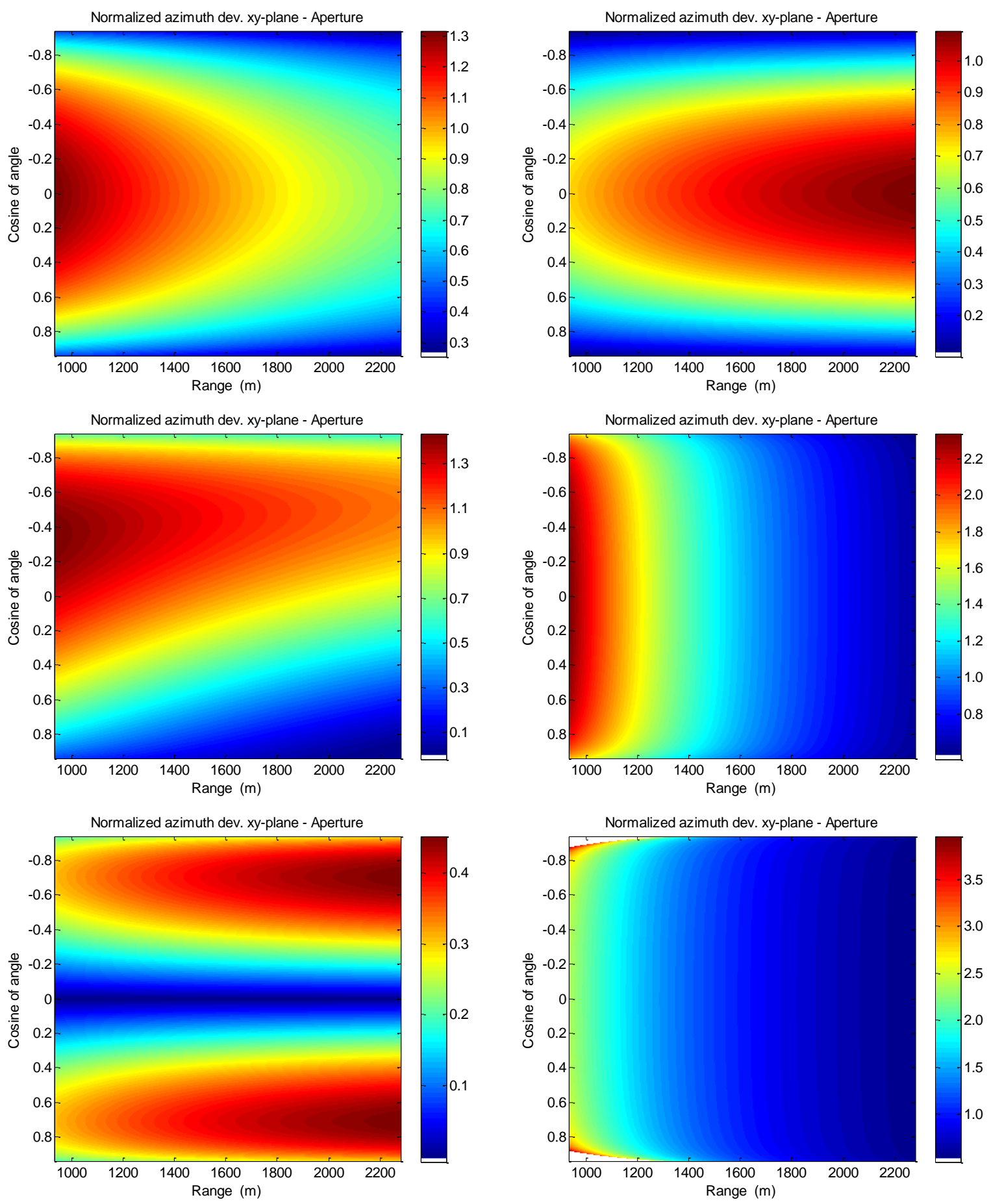

Figure 3 shows sensitivity plots or $\varepsilon_{\alpha}$-values for the final factorization step, i.e. for a faulty along-track velocity $\left(\Delta v_{x}=+0.052 \mathrm{~m} / \mathrm{s}\right)$, a faulty along-track acceleration $\left[\Delta a_{x}=+0.0052 \mathrm{~m} / \mathrm{s}^{2}\right.$ (two time bases)], a faulty cross-track acceleration $\left(\Delta a_{y}=+0.0063 \mathrm{~m} / \mathrm{s}^{2}\right)$, a positive FTP offset $(\Delta h=+150 \mathrm{~m})$ and a negative FTP offset $(\Delta h=-150 \mathrm{~m})$. See left, top to bottom then right, top to bottom. 

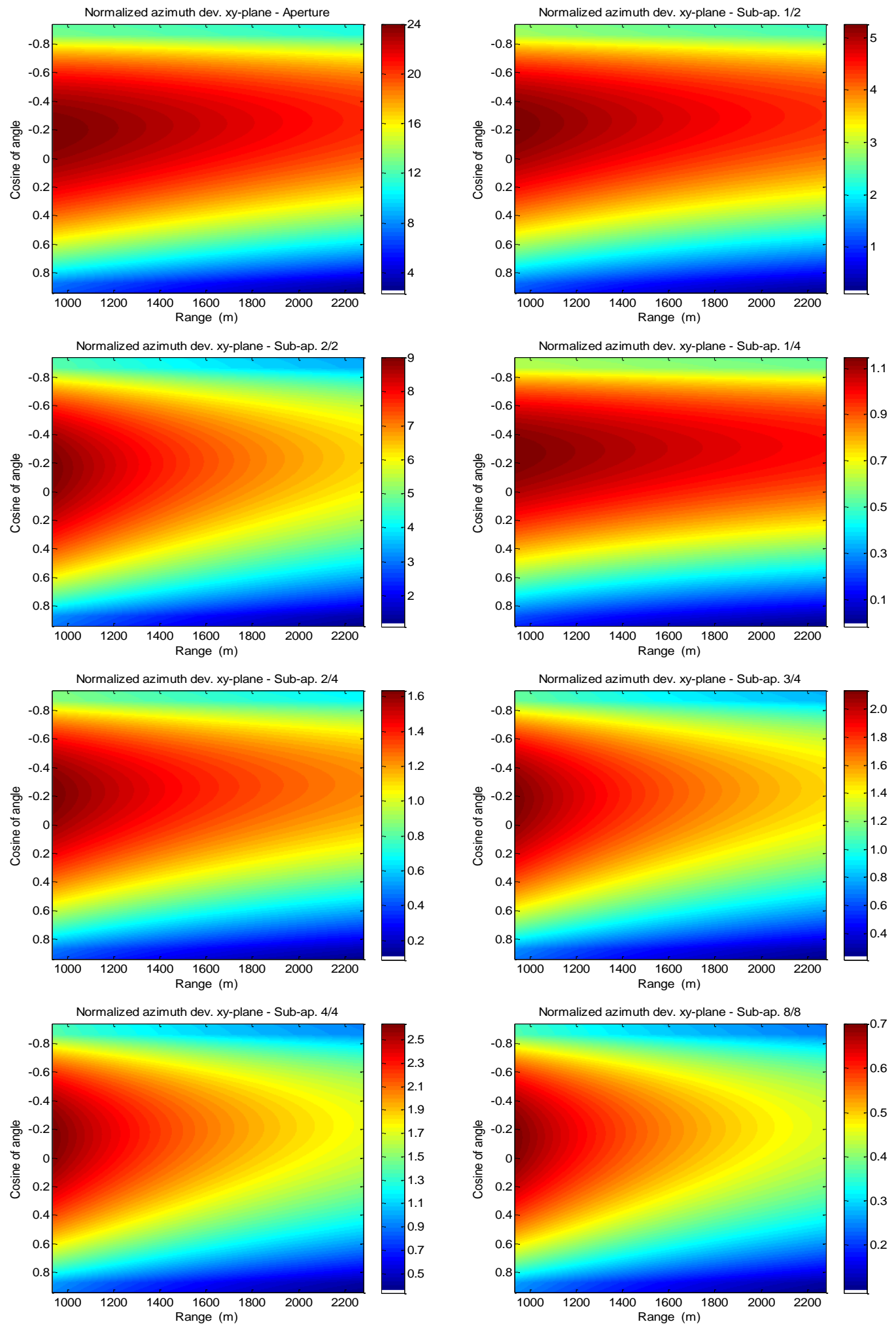

Figure 4 shows sensitivity plots or $\varepsilon_{\alpha}$-values for the three final factorization steps, plus the worst case plot for the preceding step (bottom, right), i.e. for a faulty $x y z$ acceleration $\left(\Delta a_{x}=\Delta a_{y}=\Delta a_{z}=+0.05 \mathrm{~m} / \mathrm{s}^{2}\right)$. 

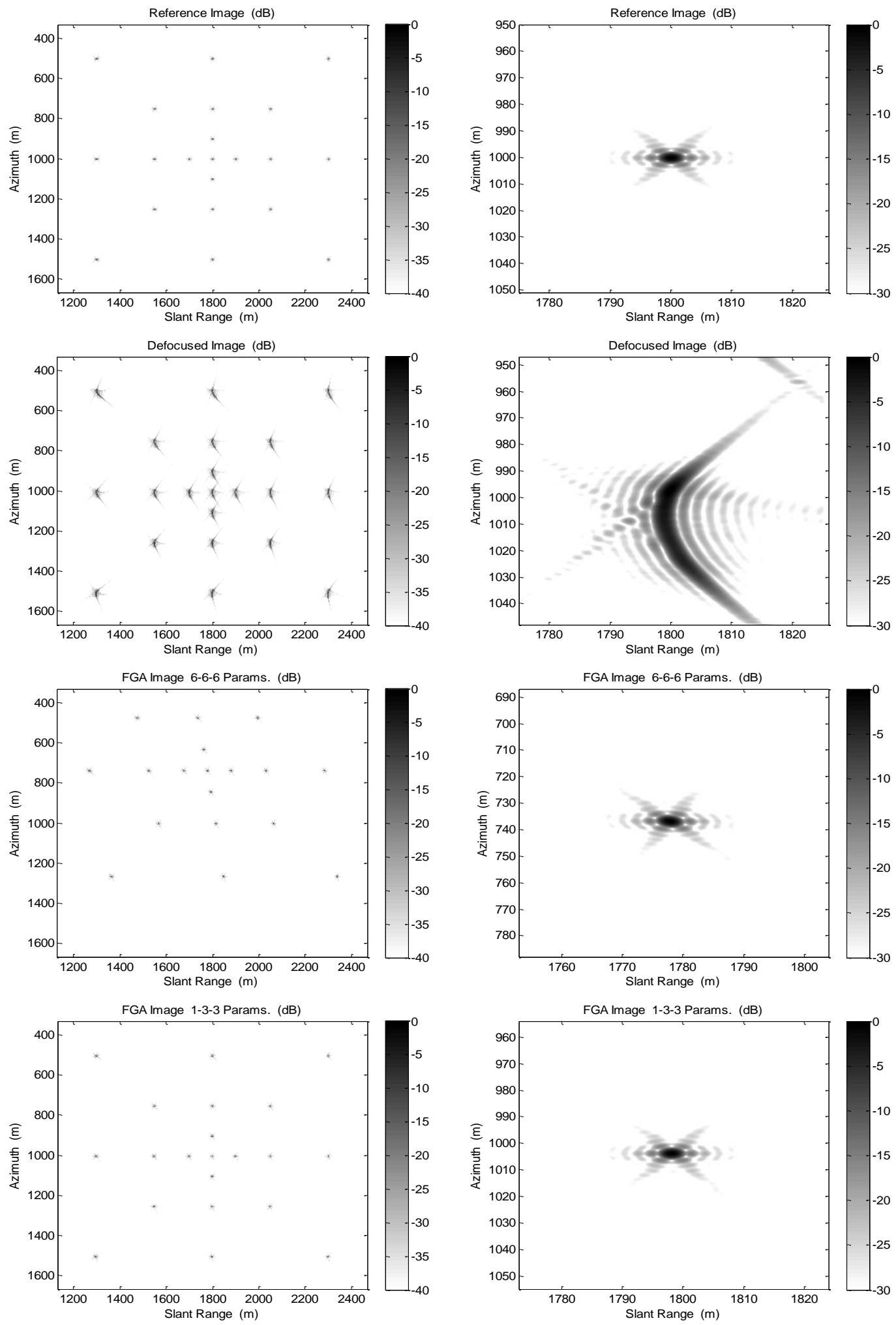

Figure 5 (left, top to bottom) Reference (focused) image, defocused image, autofocused (FGA) image (full parameter set, 6-6-6, i.e. the number of quantities adopted stage-by-stage) and FGA image (reduced parameter set, 1-3-3, i.e. the number of quantities adopted stage-by-stage). (right, top to bottom) Close-up views of the centre target, in corresponding left-side images. 